\title{
Exponential Growth of Particle Number far from the Parametric Resonance Regime
}

\author{
Fernando da Rocha Vaz Bandeira de Melo \\ Instituto de Física Gleb Wataghin, Univesidade Estadual de Campinas, Campinas, SP, Brazil \\ Robert H. Brandenberger \\ Physics Department, Brown University, Providence, RI, 02912, USA \\ and \\ Theory Division, CERN, CH-1211 Genève 23, Switzerland \\ Adolfo Maia Junior \\ Instituto de Matemática, Estatística e Computação Científica, Universidade Estadual de Campinas, Campinas, SP, Brazil
}

(February 11, 2014)

\begin{abstract}
Parametric resonance has received a considerable amount of interest as a good mathematical model to describe the initial stages of the reheating phase (matter creation) in inflationary cosmology. It is also known that exponential particle creation can occur in situations which do not fall in the parametric resonance regime characterized by oscillations of the inflaton field about its minimum. Here we present a new analytical approach to exponential particle production which can occur when the inflaton is far from the minimum of its potential. Crucial for this effect is a term in the equation of motion which acts like a negative mass square term, as occurs for tachyonic preheating and negative coupling particle production. Our techniques apply in models with a strong coupling between matter fields $\chi$ and the inflaton $\phi$, or in some models in which the inflaton has a large amplitude of oscillation. Note that our analysis yields results which are quite model dependent. Exponential growth occurs in a model with interaction Lagrangian $-g M_{p l} \phi \chi^{2}$. However, for the interaction Lagrangian $-g^{2} \phi^{2} \chi^{2}$, our formalism shows that in the large coupling limit there can only be exponential particle production when $\phi$ crosses 0 .
\end{abstract}

\section{INTRODUCTION}

Inflationary Cosmology is currently the most widely accepted model of the very early Universe. Inflation is expected to end in a period in which the inflaton field $\phi$ is evolving rapidly and, finally, oscillating about the ground state of its potential. During this phase - commonly called the reheating phase -, energy is transferred from the inflaton field to ordinary matter, here modelled by another scalar field $\chi$, via processes which depend on the coupling between $\phi$ and $\chi$ in the interaction Lagrangian. The details of this energy transfer are important for several key issues in cosmology, among them baryogenesis, the relic gravitino abundance, and the possible production of topological defects. For example, in order for the GUT-scale baryogenesis mechanism to be effective, the temperature of matter after reheating has to be comparable or higher than the GUT scale $T=10^{16} \mathrm{GeV}$ (for recent reviews see e.g. [1] ). If the reheating process is slow (i.e. the decay rate of the inflaton is small compared to the Hubble expansion rate at the end of inflation), as is predicted using a perturbative analysis of inflaton decay [2.3], then the temperature after reheating is predicted to be much lower than the GUT scale.

Recently, however, it was realized 四 that in many models of inflation the energy transfer takes place much more rapidly, based on the phenomenon of parametric resonance. The basic idea is the following: while the inflaton field is oscillating about its minimum, it induces a periodically varying time dependence in the mass of the matter field, which in turn leads to exponential growth of the matter fields which corresponds to an explosive creation of particles. The theory was put on a firm mathematical basis in [5.6] (these analyses included the expansion of the Universe), and a comprehensive and detailed study was given in [7]. According to the new picture, the whole reheating process is divided into two phases. The first one is the phase of parametric resonance, called preheating [5]. This is an out-of-equilibrium process during which the growth of the number of particles is exponential but only occurs for momenta in certain resonance bands. In the second phase, interactions between the matter particles and back reaction effects become crucial and lead to thermalization of matter $[8]$. Although parametric resonance is an attractive mechanism for particle production, there are other possibilities to obtain exponential particle production, for example instant preheating [9], chaotic preheating 10 and tachyonic preheating 11. 12. In instant preheating, particles are produced exponentially when the inflaton field $\phi$ crosses the minimum value and the interaction potential is negative (see also 113 for a discussion of negative coupling instability), in chaotic preheating the exponential increase in the perturbations is driven by the chaotic dynamics of the back- 
ground, and tachyonic preheating is particle production driven by a negative effective mass square term which occurs during spinodal decomposition in models of new and hybrid inflation [14].

In this paper we present a new mathematical approach to exponential particle production at the end of inflation but far from the parametric resonance regime. Our effect is generated by a mass square term for the matter fields which is negative for certain periods in the evolution of the inflaton field (in this respect the mechanism is exactly what happens in tachyonic [11,12] and negative coupling 13 preheating). However, our analytical method applies also for quite general nonlinear correction terms in the equations of motion. Note that we are not assuming periodic variation of the external field. Typically, our mechanism operates for large values of $|\phi|$ but only for particular forms of the interaction Lagrangian (in particular we need a large effective coupling constant). We derive our effect making use of some results on the asymptotics of solutions of second order ordinary differential equations 15 .

In next section we summarize the relevant mathematical results quoted from [15]. In Section III we apply them to differential equations which describe the evolution of the matter fields during reheating in the presence of a dynamical inflaton field. Finally, we briefly discuss the results and point out analogies and differences with previous work.

\section{MATHEMATICAL APPROACH}

The results below are based on the asymptotic analysis of second order differential equations as described e.g. in reference [15.

We consider the ordinary differential equation

$$
\ddot{y}-p(t, \sigma) y=0,
$$

where $p(t, \sigma)$ is a real function for values of $t$ in some interval $I$, and for values of $\sigma$ in an interval $E$. The derivatives are with respect to $t$. Both $t$ and $\sigma$ are real variables, and in our applications to cosmology $t$ will be time, whereas $\sigma$ is some external parameter. In this section we recall some results concerning the behavior of the solutions $y_{1}(t)$ and $y_{2}(t)$ of (1) for values of $t$ in $I$ when $\sigma \rightarrow+\infty$.

We assume that in the limit $\sigma \rightarrow+\infty$, the function $p(t, \sigma)$ can be expanded in the following way:

$$
p(t, \sigma) \approx \sigma^{r} \sum_{n=0}^{\infty} p_{n}(t) \sigma^{-\frac{r n}{2}}
$$

where $r$ is a positive rational number. We work in subintervals $I^{\prime} \subset I$ where $p_{0}(t)$ does not vanish, but could have at most double poles in $I^{\prime}$.

With these hypotheses, by a theorem in reference [15], it can be shown that: a) In subintervals $I^{\prime} \subset I$ where $p_{0}(t)>0$, and when $\sigma \rightarrow \infty$ :

$$
\begin{aligned}
y_{1}(t)= & \left\{p_{0}(t)\right\}^{-1 / 4} \exp \left\{\int ^ { t } \left[\sigma^{r / 2}\left(p_{0}\left(t^{\prime}\right)\right)^{1 / 2}+\right.\right. \\
& \left.\left.\frac{1}{2} p_{1}\left(t^{\prime}\right)\left(p_{0}\left(t^{\prime}\right)\right)^{-1 / 2}\right] d t^{\prime}\right\}\left\{1+O\left(\frac{1}{\sigma}\right)\right\}
\end{aligned}
$$

and

$$
\begin{aligned}
y_{2}(t)= & \left\{p_{0}(t)\right\}^{-1 / 4} \exp \left\{-\int^{t}\left[\sigma^{r / 2}\left(p_{0}\left(t^{\prime}\right)\right)^{1 / 2}+\right.\right. \\
& \left.\left.\frac{1}{2} p_{1}\left(t^{\prime}\right)\left(p_{0}\left(t^{\prime}\right)\right)^{-1 / 2}\right] d t^{\prime}\right\}\left\{1+O\left(\frac{1}{\sigma}\right)\right\}
\end{aligned}
$$

b) In subintervals $I^{\prime} \subset I$ where $p_{0}(t)<0$, and when $\sigma \rightarrow \infty$ :

$$
\begin{aligned}
y_{1}(t)= & c\left\{-p_{0}(t)\right\}^{-1 / 4} \sin \left\{\int ^ { t } \left[\sigma^{r / 2}\left(-p_{0}\left(t^{\prime}\right)\right)^{1 / 2}+\right.\right. \\
& \left.\left.\frac{1}{2} p_{1}\left(t^{\prime}\right)\left(-p_{0}\left(t^{\prime}\right)\right)^{-1 / 2}+a\right] d t^{\prime}\right\}\left\{1+O\left(\frac{1}{\sigma}\right)\right\}
\end{aligned}
$$

and;

$$
\begin{aligned}
y_{2}(t)= & c\left\{-p_{0}(t)\right\}^{-1 / 4} \cos \left\{\int ^ { t } \left[\sigma^{r / 2}\left(-p_{0}\left(t^{\prime}\right)\right)^{1 / 2}+\right.\right. \\
& \left.\left.\frac{1}{2} p_{1}\left(t^{\prime}\right)\left(-p_{0}\left(t^{\prime}\right)\right)^{-1 / 2}+a\right] d t^{\prime}\right\}\left\{1+O\left(\frac{1}{\sigma}\right)\right\} .
\end{aligned}
$$

Here, the phase angle $a$ is a real constant, and $c$ is a possibly complex constant.

Thus, if $p_{0}(t)>0$ on $I^{\prime}$, in the asymptotic approximation $y(t)$ increases (or decreases) exponentially. However, if $p_{0}(t)<0$, we obtain oscillating solutions. This theorem generalizes the trivial dynamics of a harmonic oscillator with positive or negative square mass to the case where the coefficient representing the square mass is not constant in time (but does not cross zero, either). Thus, in the adiabatic limit in which the mass varies only slowly, the results are easy to understand from the point of view of physics. These results will be used in the next section in order to study exponential growth of particle number at the end of inflationary phase of the evolution of the Universe.

\section{APPLICATIONS}

In this section we use the above theorem to get some information on particle creation induced by the inflaton field. In order to be as general as possible, we do not prescribe any specific equation of motion for the inflaton field, but only consider some general properties needed, which are shared by several models of chaotic inflation. As indicated, in the following $\phi$ denotes the inflaton, and $\chi$ a scalar matter field. As in most studies of preheating, we will neglect the bare mass and self-interactions 
of the $\chi$ field. We will also neglect the expansion of the Universe. As long as the time scale for exponential increase obtained under this approximation is smaller than the Hubble expansion time, we expect the approximation to be a good one. As interaction Lagrangian we take $L_{\text {int }}=-\frac{1}{2} f(\phi) \chi^{2}$, where the function $f$ contains a large parameter which plays the role of $\sigma$ in the previous section. This parameter can be a coupling constant - it can also be the amplitude of the inflaton during a period of oscillation.

The equation of motion of the k-th Fourier mode of the scalar matter field $\chi$ is given by :

$$
\ddot{\chi}_{k}-\left(-k^{2}-f(\phi)\right) \chi_{k}=0 .
$$

Comparing this with equation (11) we see that:

$$
p(\phi)=-k^{2}-f(\phi) \text {. }
$$

As mentioned above, we can write $f(\phi)=\sigma h(\phi)$, where $\sigma$ is a large parameter. Then we can write the above equation as

$$
p(\phi)=-k^{2}-\sigma h(\phi) .
$$

Comparing with the expansion (2) we get

$$
\begin{aligned}
& p_{0}(t)=-\sigma h(\phi(t)) ; \\
& p_{2}(t)=-k^{2} ; \\
& p_{n}(t)=0 ; n \neq 0,2
\end{aligned}
$$

Now, from the key theorem in section II it follows that if the parameter $\sigma$ is large, then in time intervals where $h(\phi(t))$ is positive the solutions exhibit exponential growth, whereas in those during which $h(\phi(t))$ is negative we get bounded oscillating solutions.

In order to connect the above results with the physics of known models we now specialize to two cases:

\section{A. Case $f(\phi)=g^{2} \phi^{2}$}

In this case we can write $p(\phi)$ as

$$
p(\phi)=-k^{2}-g^{2} \phi_{0}^{2} \Phi^{2}(t)
$$

where $\Phi=\frac{\phi(t)}{\phi_{0}}$ and $\phi_{0}$ is the amplitude of the inflaton field at the beginning of the oscillatory phase during which parametric resonance occurs. Taking $\sigma=g^{2} \phi_{0}^{2}$ and $r=1$ in the expansion (2) we have:

$$
\begin{aligned}
& p_{0}=-\Phi^{2}(t) ; \\
& p_{2}=-k^{2} ; \\
& p_{n}=0 ; n \neq 0,2
\end{aligned}
$$

Since in this case $p_{0}(t)<0$ for all $t$, the solutions $\chi_{k}$ are always asymptotic oscillating when $\sigma$ is big enough. Thus, in this model no particle production occurs during time intervals when $\phi \neq 0$. This result sheds new light on the observations [7,9] that particle production is concentrated at times when $\phi=0$.

\section{B. Case $f(\phi)=g M_{p l} \phi$}

In this case:

$$
p(\phi)=-k^{2}-g M_{p l} \phi(t) .
$$

We assume that the potential can be well approximated as quadratic near the origin, and as linear for large values of $|\phi|\left(|\phi|>M_{p l}\right)$. The second assumption is made in order to obtain slow-rolling of $\phi$ for large values of $|\phi|$. In an improved analysis the slow-rolling phase would be generated by the expansion of the Universe and the resulting Hubble damping term in the scalar field equation of motion. Since $\phi_{0}$ is of the order $M_{p l}$, the condition to apply the theorem of Section (III) (to obtain large $\sigma$ ) is $k^{2}, m^{2} \ll g M_{p l} \phi_{0}$, where $m$ is the inflaton mass. Thus, in particular,

$$
g \gg\left(\frac{m}{M_{p l}}\right)^{2}
$$

For a potential of the form $V(\phi)=m^{2} \phi^{2}+\lambda \phi^{4}$ the COBE constraints on the fluctuations generated during inflation give $m / M_{p l}<10^{-6}$ and $\lambda<10^{-12}$. Thus, (14) becomes $g \gg 10^{-12}$. On the other hand, by naturalness, $g \leq \sqrt{\lambda}$. Therefore, the large coupling asymptotic analysis is valid in a large interesting region of values of $\mathrm{g}$ from $10^{-12}$ to $10^{-6}$.

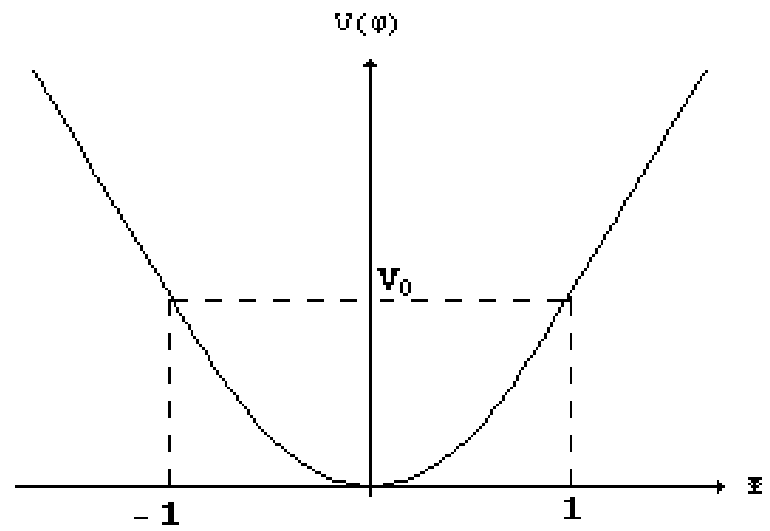

FIG. 1. Sketch of a chaotic inflation potential $V(\phi)$. $|\Phi|>1$ is the slow rolling phase in which a linear approximation to $V(\phi)$ is used. The region $|\Phi|<1$ is the region of oscillation with a quadratic approximation.

Taking $\sigma=g M_{p l} \phi_{0}$ and $r=1$ in the expansion (2), and using the same variable $\Phi$ introduced in the previous subsection, we have from (13):

$$
\begin{aligned}
& p_{0}(t)=-\Phi(t) \\
& p_{2}=-k^{2} \\
& p_{n}=0 ; n \neq 0,2 .
\end{aligned}
$$

It follows immediately that for $\Phi<0$ we have $p_{0}(t)>0$, and by the theorem presented in Section II we expect exponential particle production. 
In the following we assume that $\phi$ starts out at large negative values in the slow-rolling phase. Once it reaches $|\Phi| \sim 1, \phi$ will proceed to oscillate about the minimum of the potential. Note that exponential increase in $\chi$ is possible in both regions, as long as $\phi<0$. In the following we analyse the two phases separately. In both phases, the equation of motion is

$$
\frac{d^{2} \phi}{d t^{2}}+\frac{\partial V(\phi)}{\partial \phi}=0
$$

\section{Slow rolling region}

In the slow rolling phase we take a linear approximation to the potential, i.e., $V(\phi)=A \phi+B$, where $A$ and $B$ are constants. In the region $\phi<-\phi_{0}$, the constant $A$ is negative, and $B$ is chosen such that the potential is continuous at the transition points $|\Phi|=1$.

Solving Equation (16) in this approximation gives:

$$
\phi(t)=\frac{|A|}{2} t^{2}+C t+D
$$

where $C$ and $D<0$ are constants. Thus,

$$
p(\phi)=-k^{2}-g^{2} \frac{|A|}{2} t^{2}-g^{2} C t-g^{2} D .
$$

Taking $\sigma=g^{2}|A| / 2$ and $r=1$ in the expansion (2), we obtain from $(18)$ :

$$
\begin{aligned}
& p_{0}(t)=-\left(t^{2}+\bar{C} t+\bar{D}\right) ; \\
& p_{2}=-k^{2} ; \\
& p_{n}=0 ; n \neq 0,2
\end{aligned}
$$

where $\bar{C}=2 C /|A|$ and $\bar{D}=2 D /|A|$.

There are two roots $t_{-}$and $t_{+}$of the equation $p_{0}=0$ :

$$
t_{ \pm}=\frac{-\bar{C} \pm \sqrt{\bar{C}^{2}-4 \bar{D}}}{2}
$$

Since $D<0$, the roots are real. Thus, there is a time interval $\left[t_{-}, t_{+}\right]$during which the solution for $\chi_{k}$ is asymptotically exponential. In fact it is easy to see that $\bar{C} \leq 0$ and $\Delta t=t_{+}-t_{-} \geq|\bar{C}|=-\bar{C}$. From this inequality we conclude that the smaller $A$ or the bigger $C$, the bigger is the time interval during which the solution has exponential growth. The case of $A$ small suggests that chaotic inflation, with its long slow roll-over section of the potential, is the most effective for particle production, when the system is far from the parametric resonance regime..

Note that in the slow rolling region with $\phi>\phi_{0}, p_{0}(t)$ is negative and, as expected, we obtain asymptotically oscillating solutions.

\section{Oscillatory region}

For $|\Phi|<1$ we take a quadratic approximation, that is, $V(\phi)=A^{\prime} \phi^{2}$, where $A^{\prime}>0$. Solving the differential equation (16) for $\phi$ we get $\phi(t)=\phi_{0} \cos (w t)$, where $\phi_{0}$ is the amplitude of oscillation and $w=\sqrt{2 A}$. Thus we have:

$$
p=-k^{2}-g M_{p l} \phi_{0} \cos (w t)
$$

Taking again $\sigma=g M_{p l} \phi_{0}$ and $r=1$ in the expansion (2) we obtain from (21)

$$
\begin{aligned}
& p_{0}=-\cos (w t) ; \\
& p_{2}=-k^{2} ; \\
& p_{n}=0 ; n \neq 0,2
\end{aligned}
$$

For $\left(j-\frac{1}{2}\right) \pi<w t<\left(j+\frac{1}{2}\right) \pi$ with $j$ even, we have $p_{0}<0$. So if $\sigma$ is large, which can occur either if the coupling constant or the field amplitude are large, the theorem of Section II give us an oscillating asymptotic solution:

$$
\chi_{k}(t)=\frac{c}{\sqrt[4]{\cos (w t)}} \cos \left\{\int^{t}\left[\sigma^{1 / 2} \sqrt{\cos \left(w t^{\prime}\right)}+a\right] d t^{\prime}\right\}
$$

where $a$ and $c$ are constants.

However, for time intervals with $\left(j-\frac{1}{2}\right) \pi<w t<$ $\left(j+\frac{1}{2}\right) \pi$ with $j$ odd, we get $p_{0}>0$. Then, for large $\sigma$, the asymptotic solution reads

$$
\chi_{k}=\frac{1}{\sqrt[4]{-\cos (w t)}} e^{\sigma^{1 / 2} \int^{t} \sqrt{-\cos \left(w t^{\prime}\right)} d t^{\prime}},
$$

and in this case we get exponential production of matter.

Combining both cases, we get the time evolution of the particle number density $n_{k}$ drawn in Figure (2). Here, $n_{k}$ is the number density of particles produced in the $\mathrm{k}$-th mode obtained using the standard formula (see e.g. [7])

$$
n_{k}=w_{k}\left(\frac{\left|\dot{\chi}_{k}\right|^{2}}{w_{k}^{2}}+\left|\chi_{k}\right|^{2}\right)-\frac{1}{2}
$$

where $w_{k}$ is the effective frequency of $\chi_{k}$. Substituting (24) and (25) in (26) within their respective intervals of validity, we obtain (as a function of time) a kind of pulsed particle creation (see Fig. 2). Note that our results in this region are similar to what was previously obtained in the context of tachyonic preheating [12].

\section{DISCUSSION AND CONCLUSION}

We have presented a new analytical approach to exponential particle production far from the parametric resonance regime. Our analysis applies to situations when 


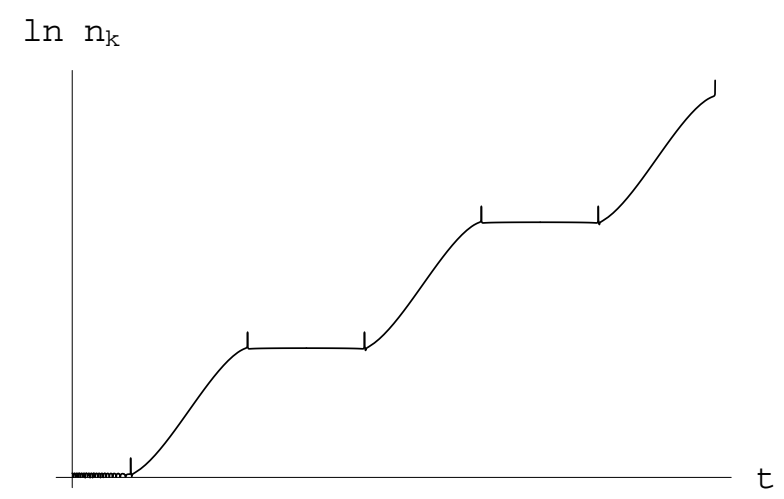

FIG. 2. Pulsed Particle Creation

the either the absolute value of the inflaton field is large, or the coupling constants are very large. The results, however, are is quite model-specific. One obtains exponential particle production for the interaction Lagrangian $L_{i n t}=-g M_{p l} \phi \chi^{2}$, but not for $L_{i n t}=-g^{2} \phi^{2} \chi^{2}$. Crucial to have exponential particle production is the presence of a negative mass square term in the effective potential for the inflaton. Note that our method is robust in the sense that the results do not change qualitatively when adding extra terms in the equation of motion such as explicit mass terms and self interaction terms for the $\chi$ field, as long as the additional terms are small in amplitude in the appropriate units.

In the rolling phase we can have exponential particle production when the coupling constant or the slope of the potential are large. However, the length of the time interval where this exponential growth happens decreases when the slope $A$ of the linear potential $V(\phi)=A \phi+$ $B$ increases. Then, when $A$ is large enough to use the asymptotic approximation, we do not have exponential matter creation for a long time. On the other hand, if $A$ is small (yielding a large interval of exponential growth), we only can use the asymptotic approximation provided the coupling constant $g$ is big enough. In this case we can have particle creation for a long time interval even during the rolling period. Applied to the regime of fast rolling, we are in the situation of tachyonic preheating, and our results agree with those of [11, 12].

\section{ACKNOWLEDGMENTS}

This work was supported in part by FAPESP, Process 99/10152/3, and by the US Department of Energy under Contract DE-FG02-91ER40688, Task A. We are grateful to Juan Garcia-Bellido, Lev Kofman and Andrei Linde for comments on the draft.
[1] A. D. Dolgov, Phys. Rept. 222, 309 (1992); A. Riotto and M. Trodden, Ann. Rev. Nucl. Part. Sci. 49, 35 (1999) hep-ph/9901362.

[2] A. D. Dolgov and A. D. Linde, Phys. Lett. B 116, 329 (1982).

[3] L. F. Abbott, E. Farhi and M. B. Wise, Phys. Lett. B 117, 29 (1982).

[4] J. H. Traschen and R. H. Brandenberger, Phys. Rev. D 42, 2491 (1990).

[5] L. Kofman, A. D. Linde and A. A. Starobinsky, Phys. Rev. Lett. 73, 3195 (1994) hep-th/9405187.

[6] Y. Shtanov, J. Traschen and R. H. Brandenberger, Phys. Rev. D 51, 5438 (1995) hep-ph/9407247.

[7] L. Kofman, A. D. Linde and A. A. Starobinsky, Phys. Rev. D 56, 3258 (1997) hep-ph/9704452.

[8] G. Felder and L. Kofman, Phys. Rev. D 63, 103503 (2001) hep-ph/0011160.

[9] G. Felder, L. Kofman and A. D. Linde, Phys. Rev. D 59, 123523 (1999) hep-ph/9812289.

[10] J. P. Zibin, "Dynamical chaos and the growth of cosmological fluctuations," hep-ph/0108008.

[11] G. Felder, J. Garcia-Bellido, P. B. Greene, L. Kofman, A. D. Linde and I. Tkachev, Phys. Rev. Lett. 87, 011601 (2001) hep-ph/0012142.

[12] G. Felder, L. Kofman and A. D. Linde, "Tachyonic instability and dynamics of spontaneous symmetry breaking," hep-th/0106179.

[13] B. R. Greene, T. Prokopec and T. G. Roos, Phys. Rev. D 56, 6484 (1997) hep-ph/9705357.

[14] A. D. Linde, Phys. Rev. D 49, 748 (1994) astro$\mathrm{ph} / 9307002$.

[15] W. D. Lakin and D. A. Sanchez, "Topics in Ordinary Differential Equations" (Dover Publ. Inc., New York, 1970). 